\title{
A posteriori error estimates in finite element solution of structure vibration problems with applications to acoustical fluid-structure analysis
}

\author{
A. Alonso, A. Dello Russo, V. Vampa
}

Abstract We present an a posteriori error estimator for piecewise linear finite element approximations of structure vibration problems. We prove that this estimator is equivalent to the energy norm of the error. Also, we introduce an adaptive mesh-refinement procedure based on the proposed estimator to analize fluid-structure interaction problems. Finally, numerical results for some test examples are presented which show the efficiency of the error estimator and the mesh-refinement techniques.

1

\section{Introduction}

Over the past several years there has been a great deal of research work on a posteriori error estimates and adaptive refinement for finite element solutions of second order elliptic problems.

Error estimates based on the evaluation of suitable local residuals were first introduced by Babuška and Rheinboldt (1978a). Later, these ideas were used by Verfürth (1989) in order to derive error estimators for the discretization of the Stokes equations. In 1987, Zienkiewicz and Zhu proposed a posteriori error estimators for problems in linear elasticity defined on some local averaging technique. Recently, Rannacher and Suttmeier (1997) presented weighted a posteriori error estimates for primal and mixed finite element solutions of problems in linear elasticity derived via duality arguments.

Although all these error estimators have been defined for time-independent problems, we mention that they could be employed in the elastodynamics and structural dynamics cases. In these situations, special attention has to be paid in order to choose a stable time-step integration

\section{A. Alonso, A. Dello Russo ${ }^{1}$, V. Vampa}

Departamento de Matemática. Facultad de Ciencias Exactas. Universidad Nacional de La Plata. C. C. 172, 1900 La Plata, Argentina

${ }^{1}$ Comisión de Investigaciones Científicas de la

Provincia de Buenos Aires, Argentina

Correspondence to: A. Dello Russo

The authors want to express their gratitude to Rodolfo Rodríguez for helpful discussions. This work was partially supported by SECYT through grant PIP-292. algorithm. Stable algorithms with time-step-adaptation have been developed by Ruge (1996) and by Smolinnski, Sleith and Belytschko (1996), for instance.

There are not many references about a posteriori error estimators for eigenvalue problems. In the one variable case, a posteriori error analysis for eigenvalue problems were first developed by Babuška and Rheinboldt (1978b). More recently, Verfürth (1994a) introduced an error estimator for eigenvalue problems in $n$-variables as an application of his general theory for non linear problems but, this estimator only yield a global lower bound on the error which is not useful for practical adaptive refinement.

In this paper, we present a posteriori error estimators for classical finite element approximations of an eigenvalue problem, namely the linear vibrations of elastic structures. We prove that this estimator is equivalent to the energy norm of the error up to higher order terms. The constants involved in this equivalence are independent of the corresponding eigenvalue and of the mesh size.

The interest of this result increases from its applications to fluid-structure interaction problems. Based on the proposed estimator, we introduce an adaptive mesh-refinemet procedure which is capable to detect local singularities of the eigenvectors of such coupled systems in the case of convex fluid domains.

Also we present some numerical experiments which show the efficiency of the error estimator and the meshrefinement techniques.

\section{2}

The structure vibration problem

2.1

Statement of the problem and its discretization

We consider the problem of determining the vibration modes in a continuous elastic medium.

Let $\Omega$ be a connected, bounded, poligonal domain in $R^{2}$ and let its boundary $\Gamma$ be split into two parts $\Gamma_{D}$ and $\Gamma_{N}$. We denote by $\mathbf{n}$ the unit normal vector to $\Gamma$.

P1. The eigenmodes $\mathbf{v} \neq 0$ and the eigenfrequencies $\lambda \geq 0$ are the solution of the following spectral problem:

$-\operatorname{div}(\sigma(\mathbf{v}))=\lambda \rho_{S} \mathbf{v} \quad$ in $\Omega$

$\mathbf{v}=0 \quad$ on $\Gamma_{D}$

$\sigma(\mathbf{v}) \mathbf{n}=0 \quad$ on $\Gamma_{N}$

where $\rho_{S}$ is the mass density and $\sigma$ is the stress tensor defined by 
$\sigma_{i j}(\mathbf{v})=\lambda_{s} \sum_{k=1}^{2} \epsilon_{k k}(\mathbf{v}) \delta_{i j}+2 \mu_{s} \epsilon_{i j}(\mathbf{v}), \quad i, j=1,2$.

In (2.4), $\lambda_{s}$ and $\mu_{s}$ are the Lamé coefficients and $\epsilon_{i j}(\mathbf{v})$ are the components of the linear strain tensor given by

$\epsilon_{i j}(\mathbf{v})=\frac{1}{2}\left(\frac{\partial v_{i}}{\partial x_{j}}+\frac{\partial v_{j}}{\partial x_{i}}\right)$.

We shall use the standard notation for Sobolev spaces $H^{m}(\Omega)$, their norms $\|\cdot\|_{m, \Omega}$ and seminorms $|\cdot|_{m, \Omega}$.

Let $H_{\Gamma_{D}}^{1}(\Omega):=\left\{\mathbf{v} \in\left(H^{1}(\Omega)\right)^{2}:\left.\mathbf{v}\right|_{\Gamma_{D}}=0\right\}$.

Let $a$ be the bilinear, symmetric, continuous form defined on $H_{\Gamma_{D}}^{1}(\Omega)$ by

$a(\mathbf{v}, \mathbf{w}):=\int_{\Omega} \sigma(\mathbf{v}): \epsilon(\mathbf{w})$

where

$\sigma(\mathbf{v}): \epsilon(\mathbf{w})=\sum_{i, j=1,2} \sigma_{i j}(\mathbf{v}) \epsilon_{i j}(\mathbf{w})$.

By using Korn's inequality, it is proved that $a$ is coercive and so, the energy norm $a(\cdot, \cdot)^{\frac{1}{2}}$ is equivalent to the usual Sobolev norm $\|\cdot\|_{1, \Omega}$ on $H_{\Gamma_{D}}^{1}(\Omega)$.

Let $b$ be the bilinear, symmetric, continuous form defined on $\left(L^{2}(\Omega)\right)^{2}$ by

$b(\mathbf{v}, \mathbf{w}):=\int_{\Omega} \rho_{S} \mathbf{v} \cdot \mathbf{w}$

The weak formulation of problem P1 is then:

P2. Find a real number $\lambda \geq 0$, and a function $\mathbf{v} \in H_{\Gamma_{D}}^{1}(\Omega)$, $\mathbf{v} \neq 0$, such that

$a(\mathbf{v}, \mathbf{w})=\lambda b(\mathbf{v}, \mathbf{w}), \quad \forall \mathbf{w} \in H_{\Gamma_{D}}^{1}(\Omega)$

Let $\left\{\mathscr{T}_{h}\right\}$ be a regular family of triangulations of $\Omega$ such that any two triangles in $\mathscr{T}_{h}$ share at most a vertex or an edge and we assume that, when the edge of a triangle intersects $\Gamma$ it is completely contained either in $\Gamma_{D}$ or in $\Gamma_{N}$.

Let $V_{h}:=\left\{\mathbf{v} \in H_{\Gamma_{D}}^{1}(\Omega):\left.\mathbf{v}\right|_{T} \in\left(P_{1}(T)\right)^{2}, \forall T \in \mathscr{T}_{h}\right\}$, where $P_{1}(T)$ denotes the set of polynomial functions defined on $T$ of degree not greater than 1 .

Then, the finite element discretization of problem $\mathbf{P} 2$ is:

P3. Find a real number $\lambda_{h} \geq 0$, and a function $\mathbf{v}_{h} \in V_{h}$, $\mathbf{v}_{h} \neq 0$, such that

$a\left(\mathbf{v}_{h}, \mathbf{w}\right)=\lambda_{h} b\left(\mathbf{v}_{h}, \mathbf{w}\right), \quad \forall \mathbf{w} \in V_{h}$

It is well known that the eigenfrequencies and the eigenfunctions of problem P3 converge to those of problem P2. By adapting the theory presented in Dautray and Lions (1990), we have the following results:

Theorem 2.1 Let $\lambda_{1} \leq \lambda_{2} \leq \cdots \leq \lambda_{n} \leq \cdots$ and $\lambda_{h 1} \leq \lambda_{h 2} \leq \cdots \leq \lambda_{h n}$ be the strictly positive eigenvalues of problems $\mathbf{P} 2$ and $\mathbf{P} 3$, respectively, in both cases repeated according to their multiplicities.

1) There exist $\beta \in(0,1]$ and a constant $C>0$, independent of $h$, such that, for h sufficiently small, the following error estimate holds $\left|\lambda_{j}-\lambda_{h j}\right| \leq C h^{2 \beta} \lambda_{j}, \quad j$ fixed

2) Let $\lambda_{j}$ be a simple eigenvalue. Let $\mathbf{v}_{j}$ be a $L^{2}-$ normalized associated eigenvector and let $\mathbf{v}_{h j}$ be a discrete eigenvector corresponding to the eigenvalue $\lambda_{h j}$ normalized in the same manner. Then, if $h$ is sufficiently small, there exist constants $C_{1}>0$ and $C_{2}>0$, independent of $h$, such that the following error estimates holds

$$
\begin{aligned}
\left\|\mathbf{v}_{j}-\mathbf{v}_{h j}\right\|_{1, \Omega} \leq C_{1} h^{\beta} \lambda_{j}, \quad j \text { fixed } \\
\left\|\mathbf{v}_{j}-\mathbf{v}_{h j}\right\|_{0, \Omega} \leq C_{2} h^{2 \beta} \lambda_{j}, \quad j \text { fixed }
\end{aligned}
$$

Error estimates similar to (2.12) can be proved for multiple eigenvalues too. The parameter $\beta$ in Theorem 2.1 depends on the reentrant corners of $\Gamma$, on the angles between $\Gamma_{D}$ and $\Gamma_{N}$ and the Lamé coefficients $\lambda_{s}$ and $\mu_{s}$.

\section{2}

\section{A posteriori error estimator}

In this section we introduce the error estimator and prove that it is equivalent to the energy norm of the error up to higher order terms.

Let $\mathbf{e}_{h}:=\mathbf{v}-\mathbf{v}_{h}$ denote the error in the approximation. Integrating by parts, we obtain from (2.9) and for any $\mathbf{w} \in H_{\Gamma_{D}}^{1}(\Omega)$

$$
\begin{aligned}
a\left(\mathbf{e}_{h}, \mathbf{w}\right)= & \int_{\Omega} \rho_{S}\left(\lambda \mathbf{v}-\lambda_{h} \mathbf{v}_{h}\right) \cdot \mathbf{w} \\
& +\sum_{T \in \mathscr{T}_{h}}\left(\int_{T} \lambda_{h} \rho_{S} \mathbf{v}_{h} \cdot \mathbf{w}-\int_{\partial T} \sigma\left(\mathbf{v}_{h}\right) \mathbf{n} \cdot \mathbf{w}\right)
\end{aligned}
$$

where, for each triangle $T, \mathbf{n}$ is its unit outward normal vector.

Let us call $\Gamma_{i}$ the union of all the interior edges of the triangulation $\mathscr{T}_{h}$. Given $l \subset \Gamma_{i}$, we denote by $\llbracket \sigma\left(\mathbf{v}_{h}\right) \mathbf{n} \rrbracket_{l}$ the jump of $\sigma\left(\mathbf{v}_{h}\right)$ in an arbitrary, but fixed, normal direction. Let

$$
\mathbf{J}_{\mathbf{l}}=\left\{\begin{array}{cl}
\llbracket \sigma\left(\mathbf{v}_{h}\right) \mathbf{n} \rrbracket_{l} & \text { if } l \subset \Gamma_{i} \\
2 \sigma\left(\mathbf{v}_{h}\right) \mathbf{n} & \text { if } l \subset \Gamma_{N} \\
0 & \text { if } l \subset \Gamma_{D}
\end{array}\right.
$$

With this notation we may write

$$
\begin{aligned}
a\left(\mathbf{e}_{h}, \mathbf{w}\right)= & \int_{\Omega} \rho_{S}\left(\lambda \mathbf{v}-\lambda_{h} \mathbf{v}_{h}\right) \cdot \mathbf{w} \\
& +\sum_{T \in \mathscr{T}_{h}}\left(\int_{T} \lambda_{h} \rho_{S} \mathbf{v}_{h} \cdot \mathbf{w}-\frac{1}{2} \sum_{l \subset \partial T} \int_{l} \mathbf{J}_{\mathbf{l}} \cdot \mathbf{w}\right), \\
& \forall \mathbf{w} \in H_{\Gamma_{D}}^{1}(\Omega)
\end{aligned}
$$

Now, for each $T \in \mathscr{T}_{h}$, we define the local error estimator $\eta_{T}$ by

$\eta_{T}^{2}=\left(\lambda_{h} \rho_{S}\right)^{2}\left\|\mathbf{v}_{h}\right\|_{0, T}^{2}|T|+\frac{1}{2} \sum_{l \subset \partial T}\left\|\mathbf{J}_{1}\right\|_{0, l}^{2}|l|$,

where $|T|$ and $|l|$ are the area of $T$ and the length of $l$, respectively. 
Let,

$\eta^{2}=\sum_{T \in \mathscr{T}_{h}} \eta_{T}^{2}$

Theorem 2.2 There exist positive constants, $C_{1}$ and $C_{2}$, only depending on the regularity of the mesh such that

$$
\begin{aligned}
\left\|\mathbf{e}_{h}\right\|_{1, \Omega} \leq & C_{1}\left(\eta+\rho_{S}\left\|\lambda \mathbf{v}-\lambda_{h} \mathbf{v}_{h}\right\|_{0, \Omega}\right) \\
\eta_{T} \leq C_{2}\left(\left\|\mathbf{e}_{h}\right\|_{1, \tilde{T}}\right. & \\
& \left.\quad+\rho_{S} \sum_{T^{*} \in \tilde{T}}\left\|\lambda \mathbf{v}-\lambda_{h} \mathbf{v}_{h}\right\|_{0, T^{*}}\left|T^{*}\right|^{\frac{1}{2}}\right)
\end{aligned}
$$

where $\tilde{T}=\bigcup\left\{T^{*} \in \mathscr{T}_{h}: T\right.$ and $T^{*}$ have a common edge $\}$. Proof:

(In what follows, $C$ will denote a constant independent of $h$ and $\lambda$, but not necessarily the same at each occurrence).

Let $\mathbf{w}^{I}$ be the continuous piecewise linear approximation of $\mathbf{w} \in H_{\Gamma_{D}}^{1}(\Omega)$ such that (see Clement (1975)),

$$
\begin{aligned}
& \mathbf{w}^{I}=0 \quad \text { on } \Gamma_{D} \\
& \left\|\mathbf{w}-\mathbf{w}^{I}\right\|_{0, T} \leq C|\mathbf{w}|_{1, \tilde{T}}|T|^{1 / 2} \quad \forall T \in \mathscr{T}_{h} \\
& \left\|\mathbf{w}-\mathbf{w}^{I}\right\|_{0, l} \leq C|\mathbf{w}|_{1, \tilde{T}}|l|^{1 / 2} \quad \forall l \subset \partial T
\end{aligned}
$$

We have then, $\mathbf{w}^{I} \in V_{h}$ and so, from (2.10), we can obtain

$$
\sum_{T \in \mathscr{T}_{h}}\left(\frac{1}{2} \sum_{l \subset \partial T} \int_{l} \mathbf{J}_{\mathbf{l}} \cdot \mathbf{w}^{I}-\int_{T} \lambda_{h} \rho_{S} \mathbf{v}_{h} \cdot \mathbf{w}^{I}\right)=0
$$

Combining (2.24) and (2.16), we may write

$$
\begin{aligned}
a\left(\mathbf{e}_{h}, \mathbf{w}\right)= & \int_{\Omega} \rho_{S}\left(\lambda \mathbf{v}-\lambda_{h} \mathbf{v}_{h}\right) \cdot \mathbf{w} \\
& +\sum_{T \in \mathscr{T}_{h}}\left(\int_{T} \lambda_{h} \rho_{S} \mathbf{v}_{h} \cdot\left(\mathbf{w}-\mathbf{w}^{I}\right)\right. \\
& \left.\quad-\frac{1}{2} \sum_{l \subset \partial T} \int_{l} \mathbf{J}_{\mathbf{l}} \cdot\left(\mathbf{w}-\mathbf{w}^{I}\right)\right)
\end{aligned}
$$

Now, using (2.22) and (2.23) we get

$a\left(\mathbf{e}_{h}, \mathbf{w}\right) \leq\left(\rho_{S}\left\|\lambda \mathbf{v}-\lambda_{h} \mathbf{v}_{h}\right\|_{0, \Omega}+C \eta\right)\|\mathbf{w}\|_{1, \Omega}$

Therefore, (2.19) follows from (2.26) and the coerciveness of $a$.

In order to prove inequality (2.20), we follow the techniques developed in Verfürth (1994b).

Let $T \in \mathscr{T}_{h}$ and choose $\mathbf{z}_{T} \in H_{\Gamma_{D}}^{1}(\Omega)$ such that supp $\mathbf{z}_{T} \subset \tilde{T}$ and

$\lambda_{h} \rho_{S}\left\|\mathbf{v}_{h}\right\|_{0, T}^{2}|T|=-\int_{T} \mathbf{v}_{h} \cdot \mathbf{z}_{T}$

$\frac{1}{2}\left\|\mathbf{J}_{\mathbf{l}}\right\|_{0, l}^{2}|l|=\int_{l} \mathrm{~J}_{\mathbf{l}} \cdot \mathbf{z}_{T} \quad \forall l \subset \partial T$

$0=\int_{T^{*}} \mathbf{v}_{h} \cdot \mathbf{z}_{T} \quad T^{*} \neq T, \forall T^{*} \subset \tilde{T}$

$\left|\mathbf{z}_{T}\right|_{1, T^{*}} \leq C \eta_{T} \quad \forall T^{*} \subset \tilde{T}$

The function $\mathbf{z}_{T}$ can be taken as a continuous piecewise quadratic polynomial augmented with local bubbles of degree four. We refer to Verfürth (1994b) for the details.

Then,

$$
\begin{aligned}
\eta_{T}^{2} & =-\rho_{S} \lambda_{h} \int_{T} \mathbf{v}_{h} \cdot \mathbf{z}_{T}+\sum_{l \subset \partial T} \int_{l} \mathbf{J}_{\mathbf{l}} \cdot \mathbf{z}_{T} \\
& =-\rho_{S} \int_{T} \lambda_{h} \mathbf{v}_{h} \cdot \mathbf{z}_{T}+\sum_{T^{*} \subset \tilde{T}}\left(\sum_{l \subset \partial T^{*}} \int_{l} \sigma\left(\mathbf{v}_{h}\right) \mathbf{n} \cdot \mathbf{z}_{T}\right) \\
& =-\rho_{S} \int_{T} \lambda_{h} \mathbf{v}_{h} \cdot \mathbf{z}_{T}+\sum_{T^{*} \subset \tilde{T}} \int_{T^{*}} \sigma\left(\mathbf{v}_{h}\right): \epsilon\left(\mathbf{z}_{T}\right)
\end{aligned}
$$

Now, since $\mathbf{z}_{T} \in H_{\Gamma_{D}}^{1}(\Omega)$, from (2.9) we have

$\int_{\tilde{T}} \sigma(\mathbf{v}): \epsilon\left(\mathbf{z}_{T}\right)-\int_{\tilde{T}} \lambda \rho_{S} \mathbf{v} \cdot \mathbf{z}_{T}=0$

Combining (2.29), (2.31) and (2.32), we can obtain

$$
\begin{array}{r}
\eta_{T}^{2}=\sum_{T^{*} \subset \tilde{T}}\left(\rho_{S} \int_{T^{*}}\left(\lambda \mathbf{v}-\lambda_{h} \mathbf{v}_{h}\right) \cdot \mathbf{z}_{T}\right. \\
\left.-\int_{T^{*}} \sigma\left(\mathbf{v}-\mathbf{v}_{h}\right): \epsilon\left(\mathbf{z}_{T}\right)\right)
\end{array}
$$

and using Schwartz inequality and standard estimates we get $\eta_{T}^{2} \leq C \sum_{T^{*} \subset \tilde{T}}\left(\rho_{s}\left\|\lambda \mathbf{v}-\lambda_{h} \mathbf{v}_{h}\right\|_{0, T^{*}}\left|T^{*}\right|^{\frac{1}{2}}+\left|\mathbf{e}_{h}\right|_{1, T^{*}}\right)\left|\mathbf{z}_{T}\right|_{1, T^{*}}$

Finally, (2.30) together with (2.34) yields

$\eta_{T}^{2} \leq C_{2}\left(\rho_{S} \sum_{T^{*} \subset \tilde{T}}\left\|\lambda \mathbf{v}-\lambda_{h} \mathbf{v}_{h}\right\|_{0, T^{*}}\left|T^{*}\right|^{\frac{1}{2}}+\left|\mathbf{e}_{h}\right|_{1, \tilde{T}}\right) \eta_{T}$

and the proof is concluded.

Remark. By using the a priori estimates (2.11) and (2.13) is not difficult to show that the second term of the right hand side of (2.19) is a higher order term. More precisely, we have

$\left\|\lambda \mathbf{v}-\lambda_{h} \mathbf{v}_{h}\right\|_{0, \Omega}=O\left(h^{2 \beta}\right)$

Also, the second term on the right hand side of Eq. (2.20) is a higher order term. In fact, we have

$\left\|\lambda \mathbf{v}-\lambda_{h} \mathbf{v}_{h}\right\|_{0, T^{*}} \leq\left|\lambda-\lambda_{h}\right|\|\mathbf{v}\|_{0, T^{*}}+\lambda_{h}\left\|\mathbf{v}-\mathbf{v}_{h}\right\|_{0, T^{*}}$

and observing that

$\left\|\mathbf{v}-\mathbf{v}_{h}\right\|_{0, T^{*}} \leq\left\|\mathbf{v}-\mathbf{v}_{h}\right\|_{1, \tilde{T}}$

it follows that $\sum_{T^{*} \subset \tilde{T}}\left\|\mathbf{v}-\mathbf{v}_{h}\right\|_{0, T^{*}}\left|T^{*}\right|^{\frac{1}{2}}$ is of higher order than $\left\|\mathbf{v}-\mathbf{v}_{h}\right\|_{1, \tilde{T}}$. Finally, we can use the a priori estimate (2.11) for the term in $\left|\lambda-\lambda_{h}\right|$.

\section{3 \\ The fluid-structure vibration problem}

\section{1}

Statement of the problem and its discretization

We consider now the problem of determining the vibration modes of an ideal fluid contained in a linear elastic structure. 
Let $\Omega_{F}$ and $\Omega_{S}$ be the domains occupied by the fluid and the solid, respectively, as shown in Fig. 1. We denote by $\Gamma_{I}$ the interface between the solid and the fluid, and by $v$ its unit normal vector pointing outwards $\Omega_{F}$. We assume that the exterior boundary of the solid is splitted into two parts, $\Gamma_{D}$ and $\Gamma_{N}$ and that the structure is fixed along $\Gamma_{D}$ and free of stress along $\Gamma_{N}$. Finally, let us denote by $\eta$ the unit outward normal vector along $\Gamma_{N}$.

P4. In the case of a compressible fluid, the eigenfunctions $(\mathbf{u}, \mathbf{v}, p) \neq(0,0,0)$ and the eigenfrequencies $\lambda \geq 0$ are the solution of the following spectral problem:

$\nabla p-\lambda \rho_{F} \mathbf{u}=0$ in $\Omega_{F}$

$p+\rho_{F} c^{2} \operatorname{div}(\mathbf{u})=0 \quad$ in $\Omega_{F}$

$\operatorname{div}(\sigma(\mathbf{v}))+\lambda \rho_{S} \mathbf{v}=0 \quad$ in $\Omega_{S}$

$\sigma(\mathbf{v}) v+p v=0$ on $\Gamma_{I}$

$\mathbf{u} \cdot v-\mathbf{v} \cdot v=0$ on $\Gamma_{I}$

$\sigma(\mathbf{v}) \eta=0 \quad$ on $\Gamma_{N}$

$\mathbf{v}=0 \quad$ on $\Gamma_{D}$

where $\mathbf{u}$ is the displacement vector in the fluid, $p$ is the fluid pressure, $\rho_{F}$ is the mass density of the fluid and $c$ is the acoustic speed in the fluid (see, for instance, Boujot (1987)).

Equations (3.36)-(3.42) define the so-called interior elastoacoustic problem. Several finite element methods based on displacement formulation have been introduced to approximate this problem (see Hamdi, Ousset and Verchery (1978), Chen and Taylor (1990), Bermúdez, Durán, Muschietti, Rodriguez and Solomin (1995), Bermúdez, Durán and Rodriguez (1998)). However, no a posteriori error estimators have been defined yet.

Consequently, it is not possible, in general, to derive adaptive mesh-refinement strategies.

It is known that the overall accuracy of the finite element approximations is deteriorated by local singularities arising from the reentrant corners of the domain $\Omega_{S}$. If the used meshes were not correctly refined there, much more computational effort would be necessary in order to obtain a numerical solution with a prescribed tolerance.

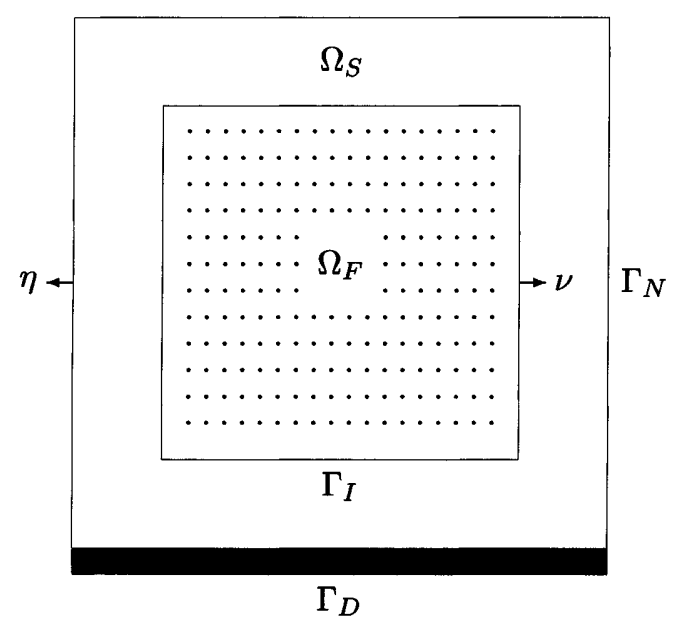

Fig. 1. Fluid and solid domains
In the next section, we will propose a simple approach to construct adequately refined meshes for coupled problems by restricting ourselves to the case of convex fluid domains.

Now, we turn our attention to the variational formulation of the coupled problem and its discretization. Here, we follow the techniques presented in Bermúdez, Durán and Rodriguez (1998) closely.

Let

$H\left(\operatorname{div}, \Omega_{F}\right):=\left\{\mathbf{u} \in L^{2}\left(\Omega_{F}\right): \operatorname{div} \mathbf{u} \in L^{2}\left(\Omega_{F}\right)\right\}$

and

$V:=H\left(\operatorname{div}, \Omega_{F}\right) \times H_{\Gamma_{D}}^{1}\left(\Omega_{S}\right)$

Multiplying Eq. (3.36) by y $\in H\left(\operatorname{div}, \Omega_{F}\right)$ and then integrating by parts and using (3.37), we obtain

$$
\begin{aligned}
& \rho_{F} c^{2} \int_{\Omega_{F}} \operatorname{div} \mathbf{u} \operatorname{div} \mathbf{y}+\int_{\Gamma_{I}} p \mathbf{y} \cdot v \\
& \quad=\lambda \rho_{F} \int_{\Omega_{F}} \mathbf{u} \cdot \mathbf{y}, \forall \mathbf{y} \in H\left(\operatorname{div}, \Omega_{F}\right)
\end{aligned}
$$

Multiplying Eq. (3.38) by $\mathbf{z} \in H_{\Gamma_{D}}^{1}\left(\Omega_{S}\right)$ and then using Green's formula and the conditions (3.39), (3.41) and (3.42), we obtain

$$
\begin{aligned}
& \int_{\Omega_{S}} \sigma(\mathbf{v}): \epsilon(\mathbf{z})-\int_{\Gamma_{I}} p \mathbf{z} \cdot v \\
& \quad=\lambda \rho_{S} \int_{\Omega_{S}} \mathbf{v} \cdot \mathbf{z}, \forall \mathbf{z} \in H_{\Gamma_{D}}^{1}\left(\Omega_{S}\right)
\end{aligned}
$$

On the other hand, because of the interface condition (3.40), for any function $\mu$ defined on $\Gamma_{I}$ we have

$\int_{\Gamma_{I}} \mu(\mathbf{u} \cdot v-\mathbf{v} \cdot v)=0$

Hence, as a consequence of (3.43), (3.44) and (3.45) we obtain the following symmetric variational eigenvalue problem:

P5. Find a real number $\lambda \geq 0$ and functions $(\mathbf{u}, \mathbf{v}, p) \in V \times L^{2}\left(\Gamma_{I}\right),(\mathbf{u}, \mathbf{v}, p) \neq(0,0,0)$, such that

$$
\begin{gathered}
\int_{\Omega_{S}} \sigma(\mathbf{v}): \epsilon(\mathbf{z})+\rho_{F} c^{2} \int_{\Omega_{F}} \operatorname{div} \mathbf{u} \operatorname{div} \mathbf{y}+\int_{\Gamma_{I}} p(\mathbf{y} \cdot v-\mathbf{z} \cdot v) \\
=\lambda\left(\int_{\Omega_{S}} \rho_{S} \mathbf{v} \cdot \mathbf{z}+\int_{\Omega_{F}} \rho_{F} \mathbf{u} \cdot \mathbf{y}\right), \forall(\mathbf{y}, \mathbf{z}) \in V \\
\int_{\Gamma_{I}} \mu(\mathbf{u} \cdot v-\mathbf{v} \cdot \mathbf{v})=0, \quad \forall \mu \in L^{2}\left(\Gamma_{I}\right)
\end{gathered}
$$

It can be proved that problems $\mathbf{P 4}$ and $\mathbf{P 5}$ are equivalent (see Bermúdez, Durán and Rodríguez (1998)).

Let us introduce the following bilinear, symmetric and continuous forms

$$
a((\mathbf{u}, \mathbf{v}),(\mathbf{y}, \mathbf{z})):=\int_{\Omega_{S}} \sigma(\mathbf{v}): \epsilon(\mathbf{z})+\rho_{F} c^{2} \int_{\Omega_{F}} \operatorname{div} \mathbf{u} \operatorname{div} \mathbf{y}
$$

$$
(\mathbf{u}, \mathbf{v}),(\mathbf{y}, \mathbf{z}) \in V
$$




$$
\begin{array}{r}
b((\mathbf{u}, \mathbf{v}),(\mathbf{y}, \mathbf{z})):=\int_{\Omega_{S}} \rho_{S} \mathbf{v} \cdot \mathbf{z}+\int_{\Omega_{F}} \rho_{F} \mathbf{u} \cdot \mathbf{y}, \\
(\mathbf{u}, \mathbf{v}),(\mathbf{y}, \mathbf{z}) \in W
\end{array}
$$

where

$$
W:=\left(L^{2}\left(\Omega_{F}\right)\right)^{2} \times\left(L^{2}\left(\Omega_{S}\right)\right)^{2}
$$

The bilinear form $a$ is not coercive on

$$
\begin{gathered}
X:=\left\{(\mathbf{u}, \mathbf{v}) \in V: \int_{\Gamma_{I}} \mu(\mathbf{u} \cdot v-\mathbf{v} \cdot v)=0,\right. \\
\left.\forall \mu \in L^{2}\left(\Gamma_{I}\right)\right\}
\end{gathered}
$$

In fact, for any $\zeta \in H_{0}^{1}\left(\Omega_{F}\right),(\operatorname{curl} \zeta, 0) \in X$ and $a((\operatorname{curl} \zeta, 0),(\operatorname{curl} \zeta, 0))=0$. However, $a^{*}=a+b$ is coercive and it can be used instead of $a$. So, we consider the following modified eigenvalue problem:

P6. Find a real number $\lambda \geq 0$ and functions $(\mathbf{u}, \mathbf{v}, p) \in V \times L^{2}\left(\Gamma_{I}\right),(\mathbf{u}, \mathbf{v}, p) \neq(0,0,0)$, such that

$$
\begin{aligned}
& a^{*}((\mathbf{u}, \mathbf{v}),(\mathbf{y}, \mathbf{z}))+\int_{\Gamma_{I}} p(\mathbf{y} \cdot v-\mathbf{z} \cdot v) \\
& \quad=(\lambda+1) b((\mathbf{u}, \mathbf{v}),(\mathbf{y}, \mathbf{z})), \forall(\mathbf{y}, \mathbf{z}) \in V \\
& \int_{\Gamma_{I}} \mu(\mathbf{u} \cdot v-\mathbf{v} \cdot \mathbf{v})=0, \quad \forall \mu \in L^{2}\left(\Gamma_{I}\right)
\end{aligned}
$$

Clearly, problem P6 has the same eigenvalues and eigenfunctions than problem P5. Moreover, following the techniques in Bermúdez, Durán and Rodríguez (1998), it can be proved that the forms $a^{*}$ on $V \times V$ and $\int_{\Gamma_{I}} \mu(\mathbf{y} \cdot v-\mathbf{z} \cdot v)$ on $V \times L^{2}\left(\Gamma_{I}\right)$ satisfy the classical Brezzi's conditions (see Brezzi and Fortin (1991)). Then, problem P6 is well posed.

Let $\left\{\mathscr{T}_{h}\right\}$ be a family of regular triangulations of $\Omega_{F} \cup \Omega_{S}$ such that every triangle is completely contained either in $\Omega_{F}$ or in $\Omega_{S}$. To discretize the displacements in the fluid we used the lowest order Raviart-Thomas finite element space (see Raviart and Thomas (1977)),

$$
\begin{aligned}
R_{h}\left(\Omega_{F}\right):= & \left\{\mathbf{u} \in H\left(\operatorname{div}, \Omega_{F}\right):\left.\mathbf{u}\right|_{T} \in R T_{0}(T),\right. \\
& \left.\forall T \in \mathscr{T}_{h}, T \subset \Omega_{F}\right\}
\end{aligned}
$$

where

$$
\begin{aligned}
R T_{0}:= & \left\{\mathbf{u} \in P_{1}(T)^{2}: \mathbf{u}(x, y)=(a+b x, c+b y)\right. \\
& : a, b, c \in R\}
\end{aligned}
$$

For each component of the displacement in the solid we use the standard finite element space

$$
\begin{gathered}
L_{h}\left(\Omega_{S}\right):=\left\{\mathbf{v} \in H^{1}\left(\Omega_{S}\right):\left.\mathbf{v}\right|_{T} \in P_{1}(T),\right. \\
\left.\forall T \in \mathscr{T}_{h}, T \subset \Omega_{S}\right\}
\end{gathered}
$$

The discrete analogue of $V$ is then

$$
V_{h}:=\left\{(\mathbf{u}, \mathbf{v}) \in R_{h}\left(\Omega_{F}\right) \times\left(L_{h}\left(\Omega_{S}\right)\right)^{2}:\left.\mathbf{v}\right|_{\Gamma_{D}}=0\right\}
$$

Finally, for the pressure in the interface we use the space of piecewise constant functions

$$
Q_{h}:=\left\{p \in L^{2}\left(\Gamma_{I}\right):\left.p\right|_{T} \in P_{0}(l), \forall l \in \Gamma_{I}\right\}
$$

Then, the finite element approximation of problem P6 is:

P7. Find a real number $\lambda_{h} \geq 0$ and functions $\left(\mathbf{u}_{h}, \mathbf{v}_{h}, p_{h}\right) \in V_{h} \times Q_{h},\left(\mathbf{u}_{h}, \mathbf{v}_{h}, p_{h}\right) \neq(0,0,0)$, such that

$$
\begin{aligned}
& a^{*}\left(\left(\mathbf{u}_{h}, \mathbf{v}_{h}\right),(\mathbf{y}, \mathbf{z})\right)+\int_{\Gamma_{I}} p_{h}(\mathbf{y} \cdot v-\mathbf{z} \cdot v) \\
& \quad=\left(\lambda_{h}+1\right) b\left(\left(\mathbf{u}_{h}, \mathbf{v}_{h}\right),(\mathbf{y}, \mathbf{z})\right), \quad \forall(\mathbf{y}, \mathbf{z}) \in V_{h}
\end{aligned}
$$

$\int_{\Gamma_{I}} \mu\left(\mathbf{u}_{h} \cdot v-\mathbf{v}_{h} \cdot \mathbf{v}\right)=0, \quad \forall \mu \in Q_{h}$

Once more, following the techniques in Bermúdez, Durán and Rodríguez (1998), it can be proved that the forms $a^{*}$ and $\int_{\Gamma_{I}} \mu(\mathbf{y} \cdot v-\mathbf{z} \cdot v)$ on the discrete spaces $V_{h} \times V_{h}$ and $V_{h} \times Q_{h}$, respectively, satisfy both Brezzi's conditions uniformly in $h$.

In Bermúdez, Durán and Rodríguez (1998) and Rodríguez and Solomin (1996) it was proved that the eigenfunctions and eigenfrequencies of problem P7 converge to those of problem P6 and that non-zero frequency spurious modes do not arise in this discretization. More precisely, we have:

Theorem 3.1 Let $\lambda_{1} \leq \lambda_{2} \leq \cdots \leq \lambda_{n} \leq \cdots$ and $\lambda_{h 1} \leq \lambda_{h 2} \leq \cdots \leq \lambda_{h n}$ be the strictly positive eigenvalues of problems $\mathbf{P 6}$ and $\mathbf{P 7}$, respectively, in both cases repeated according to their multiplicities.

1) There exist $\alpha \in(0,1]$ and a constant $C>0$, independent of $h$, such that, for $h$ sufficiently small, the following error estimate holds

$\left|\lambda_{j}-\lambda_{h j}\right| \leq C h^{2 \alpha}, \quad j$ fixed

2) Let $\lambda_{j}$ be a simple eigenvalue. Let $\left(\mathbf{u}_{j}, \mathbf{v}_{j}\right)$ be a normalized associated eigenvector and let $\left(\mathbf{u}_{h j}, \mathbf{v}_{h j}\right)$ be a discrete eigenvector corresponding to the eigenvalue $\lambda_{h j}$ normalized in the same manner. Then, if $h$ is sufficiently small, there exist constants $C_{1}>0$ and $C_{2}>0$, independent of $h$, such that the following error estimates holds

$$
\begin{aligned}
& \left\|\mathbf{u}_{j}-\mathbf{u}_{h j}\right\|_{H\left(\operatorname{div}, \Omega_{F}\right)}+\left\|\mathbf{v}_{j}-\mathbf{v}_{h j}\right\|_{1, \Omega_{S}} \leq C_{1} h^{\alpha}, \\
& \qquad \text { fixed } \\
& \left\|p-p_{h}\right\|_{0, \Gamma_{I}} \leq C_{2} h^{\alpha}, \quad j \text { fixed }
\end{aligned}
$$

Similar results to those in property 2 can be proved for multiple eigenvalues too. The value of the constant $\alpha$ in (3.54), (3.55) and (3.56) depends on the regularity of the eigenmodes in both the fluid and the solid domains.

\section{2}

\section{An adaptive mesh-refinement procedure}

If $\Omega_{F}$ is convex, it is natural to suppose that the eigenfunctions corresponding to the fluid part of the system will remain almost regular. So, we may assume that the main part of the error in the approximation of the eigenvectors is related with the error in the approximation of the structure displacements.

Going back to Eq. (3.44), we can interpret it as the variational formulation of the problem of harmonic response of the structure to pressure actions of the fluid (see Morand and Ohayon (1995)). In this sense, we define the local error estimator $\eta_{T}$ by 
$\eta_{T}^{2}=\left(\lambda_{h} \rho_{S}\right)^{2}\left\|\mathbf{v}_{h}\right\|_{0, T}^{2}|T|+\frac{1}{2} \sum_{l \subset \partial T}\left\|\mathbf{J}_{1}\right\|_{0, l}^{2}|l|, \quad \forall T \subset \Omega_{S}$

where

$\mathbf{J}_{\mathbf{l}}=\left\{\begin{array}{cl}\llbracket \sigma\left(\mathbf{v}_{h}\right) \mathbf{n} \rrbracket_{l} & \text { if } l \subset \Gamma_{i} \\ 2 \sigma\left(\mathbf{v}_{h}\right) \mathbf{n} & \text { if } l \subset \Gamma_{N} \\ 2\left(\sigma\left(\mathbf{v}_{h}\right) \mathbf{n}+p_{h} \mathbf{n}\right) & \text { if } l \subset \Gamma_{I} \\ 0 & \text { if } l \subset \Gamma_{D}\end{array}\right.$

Also, we define the following adaptive mesh-refinement procedure.

Let $\mathscr{T}_{k}$ be any mesh of $\Omega_{F} \cup \Omega_{S}$; for instance, $\mathscr{T}_{k}$ may be the starting mesh. After having computed the local error estimators for each triangle $T \subset \Omega_{S}$, we are able to decide which elements of $\Omega_{S}$ have to be refined. By doing so, we can construct a new mesh for the solid domain. This step of the process may lead to introduce new nodes on the interface between the solid and the fluid. So, in order to construct a new mesh for the fluid domain, we simply require that it be compatible with the one already obtained for the solid. In this way, we can obtain the next mesh $\mathscr{T}_{k+1}$ of $\Omega_{S} \cup \Omega_{F}$.

The results of the next section show that it is possible to improve the quality of the finite element approximations in an efficient manner by using this strategy.

\section{4}

\section{Numerical results}

In this section we present numerical results for two test problems. These problems were already considered by Bermúdez and Rodriguez (1994). In order to make comparisons easier between uniform and adaptive mesh-refinement techniques, we include here the numerical results reported by these authors.

The first problem correspond to a steel cavity with vacuum inside. The geometrical data can be seen in Fig. 2 .

The values of the physical parameters are the following: $\rho_{S}=7.710^{3} \mathrm{~kg} / \mathrm{m}^{3}$

Young's modulus $=1.4410^{11} \mathrm{~Pa}$

Poisson's coefficient $=0.35$

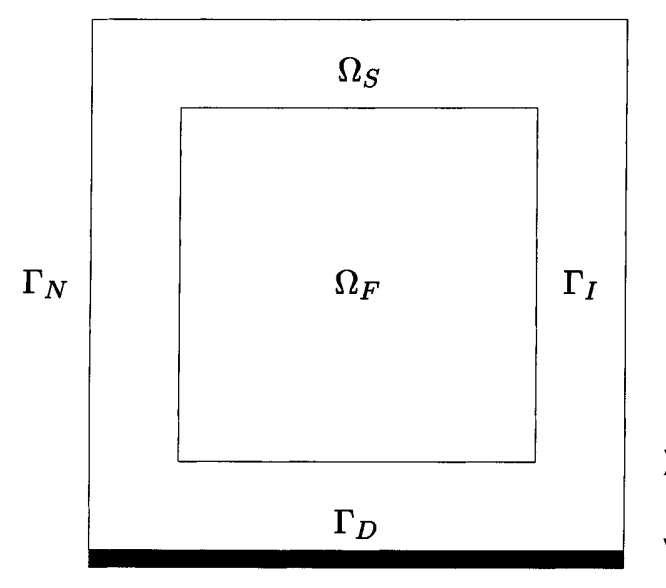

$0.25 \mathrm{~m}$

$1.00 \mathrm{~m}$

$0.25 \mathrm{~m}$
Table 1 presents the computed lowest eigenfrequencies for several embedded meshes. The parameter $N$ denotes the number of nodes. Because no analytical expression for the eigenvalues is known, the computed ones have been extrapolated to obtain what will be denoted by $\lambda_{\text {exact }}$. This extrapolation technique has also been used to get an estimation of the order of convergence in powers of $h=O\left(\frac{1}{\sqrt{N}}\right)$.

Since $\Omega_{S}$ is not convex, strong singularities arise in the vicinity of the reentrant corners. In this case, the order of convergence of the eigenvectors is less than 2 which is the order predicted by the theory for regular eigenfunctions.

We present below the results obtained with meshes generated by the following adaptive method. The process starts with a uniform triangulation $\mathscr{T}_{0}$. By using $\eta_{T}$ as an error indicator at the element $T, \mathscr{T}_{k+1}$ is obtained from $\mathscr{T}_{k}$ refining all $T \in \mathscr{T}_{k}$ with

$\eta_{T} \geq \gamma \max _{T^{\prime} \in \mathscr{T}_{k}} \eta_{T^{\prime}}$

In our experiments we have taken $\gamma=0.7$ and we have started the process from a very coarse mesh.

Table 2 to Table 5 show the results obtained in eight steps of the refinement procedure for eigenmodes $S_{1}, S_{2}, S_{3}$ and $S_{4}$, respectively. Now, the order of convergence is computed in powers of $\frac{1}{\sqrt{N}}$ and it is almost exactly 2 for all these eigenfrequencies. In another words, the optimal order of convergence with respect to the number of nodes was obtained for these eigenmodes. It is interesting to observe the significant reduction of the necessary computational effort to obtain a solution with a prescribed accuracy.

Numerical experiments for eigenmodes $S_{5}, S_{6}, S_{7}$ and $S_{8}$ were also performed. In all these cases optimal order of

Table 1. Steel cavity with vacuum inside: eigenfrequencies computed by using uniform refinement

\begin{tabular}{lrrrrr}
\hline mode & $N=1440$ & $N=5440$ & $N=21120$ & \multicolumn{1}{l}{ order } & \multicolumn{1}{l}{$\lambda_{\text {exact }}$} \\
\hline$S_{1}$ & 699.879 & 678.275 & 670.369 & 1.48 & 665.918 \\
$S_{2}$ & 2351.270 & 2305.042 & 2290.833 & 1.72 & 2284.617 \\
$S_{3}$ & 3957.305 & 3857.876 & 3822.467 & 1.51 & 3803.266 \\
$S_{4}$ & 4031.904 & 3942.979 & 3905.800 & 1.28 & 3879.595 \\
$S_{5}$ & 4548.893 & 4523.909 & 4514.040 & 1.35 & 4507.626 \\
$S_{6}$ & 5578.104 & 5505.724 & 5482.094 & 1.63 & 5470.751 \\
$S_{7}$ & 7774.507 & 7626.125 & 7583.145 & 1.81 & 7565.857 \\
$S_{8}$ & 7783.139 & 7693.529 & 7660.384 & 1.45 & 7641.107 \\
\hline
\end{tabular}

Table 2. Eigenmode $S_{1}$ computed by using adaptive refinement

\begin{tabular}{llll}
\hline $\mathrm{k}$ & $N$ & $\lambda_{h}$ & $\left|\lambda_{\text {exact }}-\lambda_{h}\right|$ \\
\hline 0 & 120 & 908.135 & 242.217 \\
1 & 154 & 790.877 & 124.959 \\
2 & 214 & 733.956 & 68.038 \\
3 & 232 & 727.204 & 61.286 \\
4 & 318 & 711.914 & 45.996 \\
5 & 494 & 695.364 & 29.446 \\
6 & 805 & 683.289 & 17.371 \\
7 & 1075 & 678.770 & 12.852 \\
8 & 1508 & 674.755 & 8.837 \\
\multicolumn{2}{l}{ order $=2.079$} & & \\
\hline
\end{tabular}

Fig. 2. Steel cavity 
Table 3. Eigenmode $S_{2}$ computed by using adaptive refinement

\begin{tabular}{llll}
\hline $\mathrm{k}$ & $N$ & $\lambda_{h}$ & $\left|\lambda_{\text {exact }}-\lambda_{h}\right|$ \\
\hline 0 & 120 & 2833.214 & 548.597 \\
1 & 199 & 2497.795 & 213.178 \\
2 & 221 & 2465.705 & 181.088 \\
3 & 245 & 2441.844 & 157.227 \\
4 & 302 & 2419.078 & 134.461 \\
5 & 502 & 2369.878 & 85.261 \\
6 & 738 & 2337.921 & 53.304 \\
7 & 1058 & 2321.863 & 37.246 \\
8 & 1274 & 2316.273 & 31.656 \\
\multicolumn{2}{l}{ order $=2.019$} & &
\end{tabular}

convergence was obtained. These results allow us to conclude that our error estimator efficiently detects the regions where the mesh must be refined.

Figure 3 shows, for eigenmode $S_{1}$, the initial triangulation together with three refined triangulations.

In the second test problem the interior $\Omega_{F}$ of the steel cavity was completely filled with water. The physical parameters of the fluid are the following:

$$
\begin{aligned}
\rho_{F} & =1000 \mathrm{~kg} / \mathrm{m}^{3} \\
c & =1430 \mathrm{~m} / \mathrm{s}
\end{aligned}
$$

Table 6 presents the computed eigenfrequencies for this fluid-structure interaction problem obtained with the finite element method described above and using two
Table 4. Eigenmode $S_{3}$ computed by using adaptive refinement

\begin{tabular}{llll}
\hline $\mathrm{k}$ & $N$ & $\lambda_{h}$ & $\left|\lambda_{\text {exact }}-\lambda_{h}\right|$ \\
\hline 0 & 120 & 4632.095 & 828.829 \\
1 & 160 & 4433.584 & 630.318 \\
2 & 228 & 4221.537 & 418.271 \\
3 & 242 & 4147.363 & 344.097 \\
4 & 304 & 4086.109 & 282.843 \\
5 & 437 & 4013.075 & 209.809 \\
6 & 728 & 3922.954 & 119.688 \\
7 & 895 & 3896.265 & 92.999 \\
8 & 1251 & 3872.500 & 69.234 \\
\multicolumn{2}{l}{ order $=2.051$} & & \\
\hline
\end{tabular}

Table 5. Eigenmode $S_{4}$ computed by using adaptive refinement

\begin{tabular}{lrll}
$\mathrm{k}$ & $N$ & $\lambda_{h}$ & $\left|\lambda_{\text {exact }}-\lambda_{h}\right|$ \\
\hline 0 & 120 & 4614.781 & 735.186 \\
1 & 142 & 4418.653 & 539.058 \\
2 & 222 & 4180.860 & 301.265 \\
3 & 274 & 4122.740 & 243.145 \\
4 & 326 & 4085.822 & 206.227 \\
5 & 531 & 4007.080 & 127.485 \\
6 & 727 & 3970.639 & 91.044 \\
7 & 1010 & 3944.757 & 65.162 \\
8 & 1448 & 3925.674 & 46.079 \\
order $=2.077$ & & \\
\hline
\end{tabular}

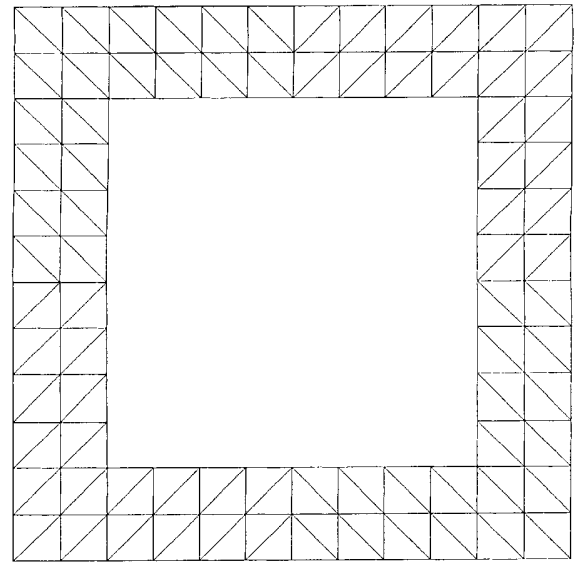

a

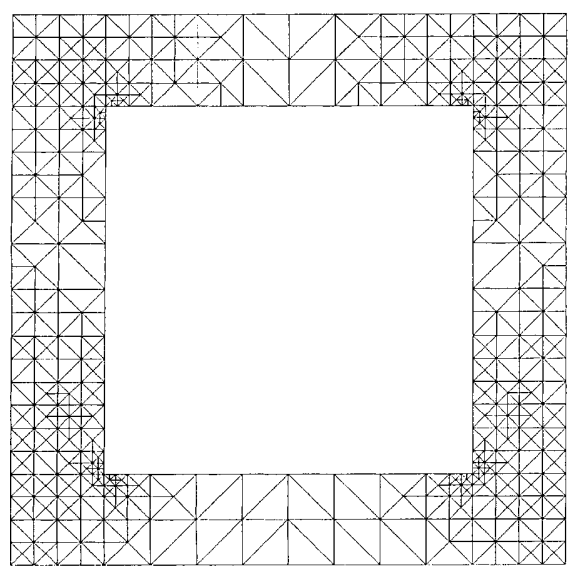

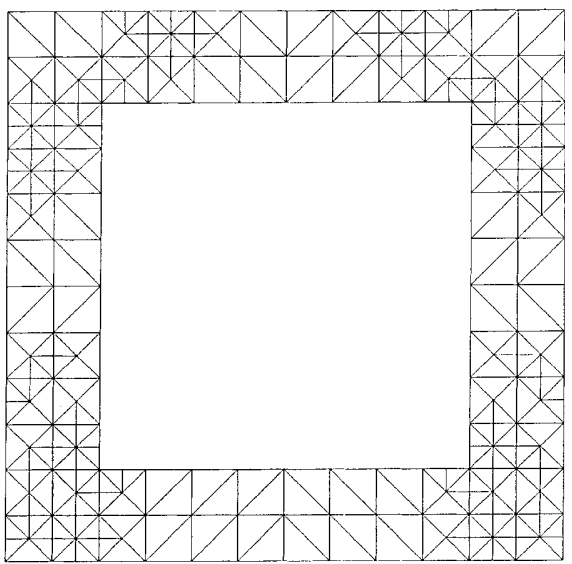

b

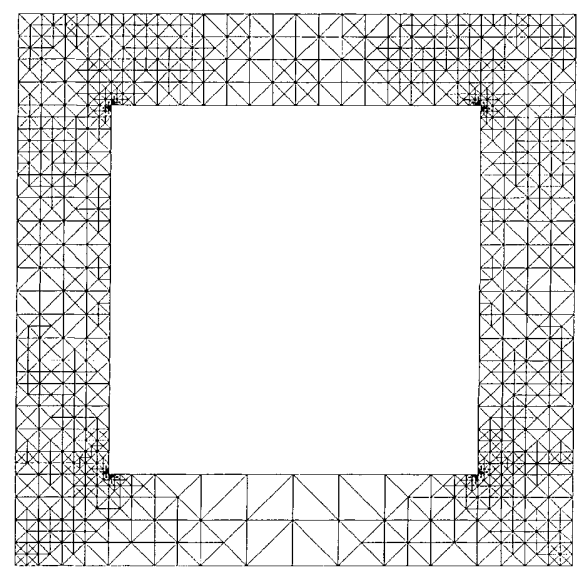

d
Fig. 3a-d. Initial triangulation and some refined triangulations for Eigenmode $S_{1}$. a Initial triangulation, $\mathbf{b}$ Third refinement step, c Fifth refinement step, d Seventh refinement step 
Table 6. Water in a steel cavity: eigenfrequencies computed by using uniform refinement

\begin{tabular}{lrrrlr}
\hline mode & $N=448$ & $N=1600$ & $N=6016$ & order & \multicolumn{1}{l}{$\lambda_{\text {exact }}$} \\
\hline$S_{1}$ & 877.390 & 734.427 & 676.262 & 1.49 & 641.837 \\
$S_{2}$ & 2641.617 & 2333.958 & 2203.241 & 1.37 & 2116.398 \\
$S_{3}$ & 4005.084 & 3714.027 & 3524.046 & 0.71 & 3201.475 \\
$S_{4}$ & 4314.221 & 4092.835 & 3965.678 & 0.86 & 3804.124 \\
$S_{5}$ & 4478.750 & 4291.165 & 4234.950 & 1.78 & 4211.620 \\
$F_{01}$ & 4921.544 & 4791.399 & 4733.413 & 1.19 & 4687.927 \\
$F_{10}$ & 5522.427 & 5271.404 & 5191.406 & 1.69 & 5155.246 \\
$S_{6}$ & 6275.157 & 5734.823 & 5519.013 & 1.42 & 5385.805 \\
$F_{11}$ & 6421.012 & 6344.273 & 6299.794 & 0.80 & 6239.332 \\
\hline
\end{tabular}

Table 7. Eigenmode $S_{1}$ computed by using adaptive refinement

\begin{tabular}{llll}
\hline $\mathrm{k}$ & $N$ & $\lambda_{h}$ & $\left|\lambda_{\text {exact }}-\lambda_{h}\right|$ \\
\hline 0 & 448 & 877.390 & 235.553 \\
1 & 576 & 764.373 & 122.536 \\
2 & 712 & 710.555 & 68.718 \\
3 & 748 & 703.910 & 62.073 \\
4 & 1002 & 689.016 & 47.179 \\
5 & 1540 & 671.907 & 30.070 \\
6 & 2311 & 660.398 & 18.561 \\
\hline
\end{tabular}

Table 8. Eigenmode $S_{2}$ computed by using adaptive refinement

\begin{tabular}{llll}
\hline $\mathrm{k}$ & $N$ & $\lambda_{h}$ & $\left|\lambda_{\text {exact }}-\lambda_{h}\right|$ \\
\hline 0 & 448 & 2641.618 & 525.220 \\
1 & 689 & 2340.560 & 224.162 \\
2 & 727 & 2325.297 & 208.899 \\
3 & 911 & 2277.752 & 161.354 \\
4 & 1227 & 2246.633 & 130.235 \\
5 & 1577 & 2217.761 & 101.363 \\
6 & 2439 & 2183.345 & 66.947 \\
\hline
\end{tabular}

Table 9. Eigenmode $S_{3}$ computed by using adaptive refinement

\begin{tabular}{lrll}
\hline $\mathrm{k}$ & $N$ & $\lambda_{h}$ & $\left|\lambda_{\text {exact }}-\lambda_{h}\right|$ \\
\hline 0 & 448 & 4005.082 & 803.607 \\
1 & 580 & 3898.919 & 697.444 \\
2 & 738 & 3738.824 & 537.349 \\
3 & 846 & 3640.368 & 438.893 \\
4 & 1034 & 3599.878 & 398.403 \\
5 & 1558 & 3532.658 & 331.187 \\
6 & 2243 & 3487.968 & 286.493 \\
\hline
\end{tabular}

Table 10. Eigenmode $F_{01}$ computed by using adaptive refinement

\begin{tabular}{lrlc}
\hline $\mathrm{k}$ & $N$ & $\lambda_{h}$ & $\left|\lambda_{\text {exact }}-\lambda_{h}\right|$ \\
\hline 0 & 448 & 4921.540 & 233.613 \\
1 & 514 & 4847.272 & 159.345 \\
2 & 746 & 4777.400 & 89.473 \\
3 & 904 & 4752.368 & 64.441 \\
4 & 1091 & 4739.578 & 51.651 \\
5 & 1543 & 4724.194 & 36.267 \\
6 & 2299 & 4710.665 & 22.738 \\
\hline
\end{tabular}

Table 11. Eigenmode $F_{10}$ computed by using adaptive refinement

\begin{tabular}{lllc}
\hline $\mathrm{k}$ & $N$ & $\lambda_{h}$ & $\left|\lambda_{\text {exact }}-\lambda_{h}\right|$ \\
\hline 0 & 448 & 5522.426 & 367.180 \\
1 & 618 & 5329.189 & 173.943 \\
2 & 766 & 5245.276 & 90.030 \\
3 & 1002 & 5226.149 & 70.903 \\
4 & 1346 & 5204.265 & 49.019 \\
5 & 1897 & 5185.295 & 30.049 \\
6 & 2567 & 5174.885 & 19.639 \\
\hline
\end{tabular}

Table 12. Eigenmode $F_{11}$ computed by using adaptive refinement

\begin{tabular}{lllc}
\hline $\mathrm{k}$ & $N$ & $\lambda_{h}$ & $\left|\lambda_{\text {exact }}-\lambda_{h}\right|$ \\
\hline 0 & 448 & 6421.010 & 181.678 \\
1 & 530 & 6391.597 & 152.265 \\
2 & 596 & 6380.270 & 140.938 \\
3 & 732 & 6353.549 & 114.217 \\
4 & 1046 & 6326.601 & 87.269 \\
5 & 1090 & 6322.934 & 83.602 \\
6 & 1438 & 6309.352 & 70.020 \\
\hline
\end{tabular}

succesive uniform refinements of the initial mesh. Here, $N$ denotes the number of unknowns. Once more, the order of convergence in powers of $h$ and the values of $\lambda_{\text {exact }}$ were obtained by extrapolation.

We observe that the computed order of convergence is less than 2 for all these eigenmodes. In particular, this order is significatively low for eigenmodes $S_{3}, S_{4}$ and $F_{11}$.

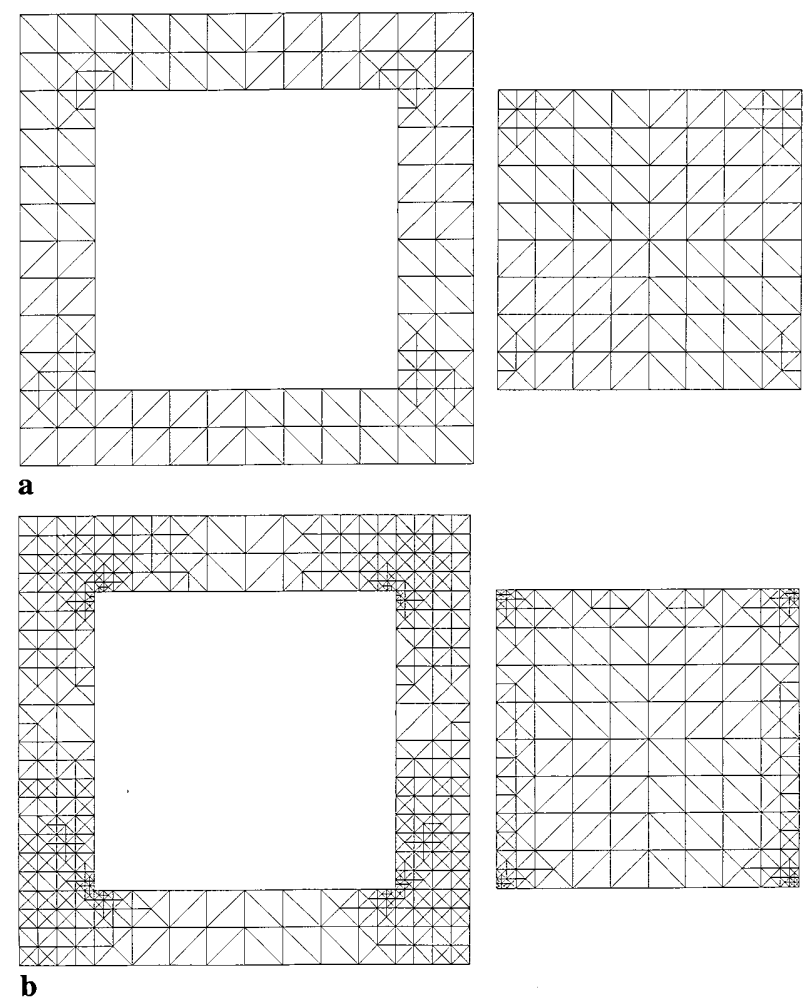

Fig. 4a,b. Some refined triangulations for Eigenmode $S_{1}$. a First refinement step for the solid and fluid domains, b Fifth refinement step for the solid and fluid domains 


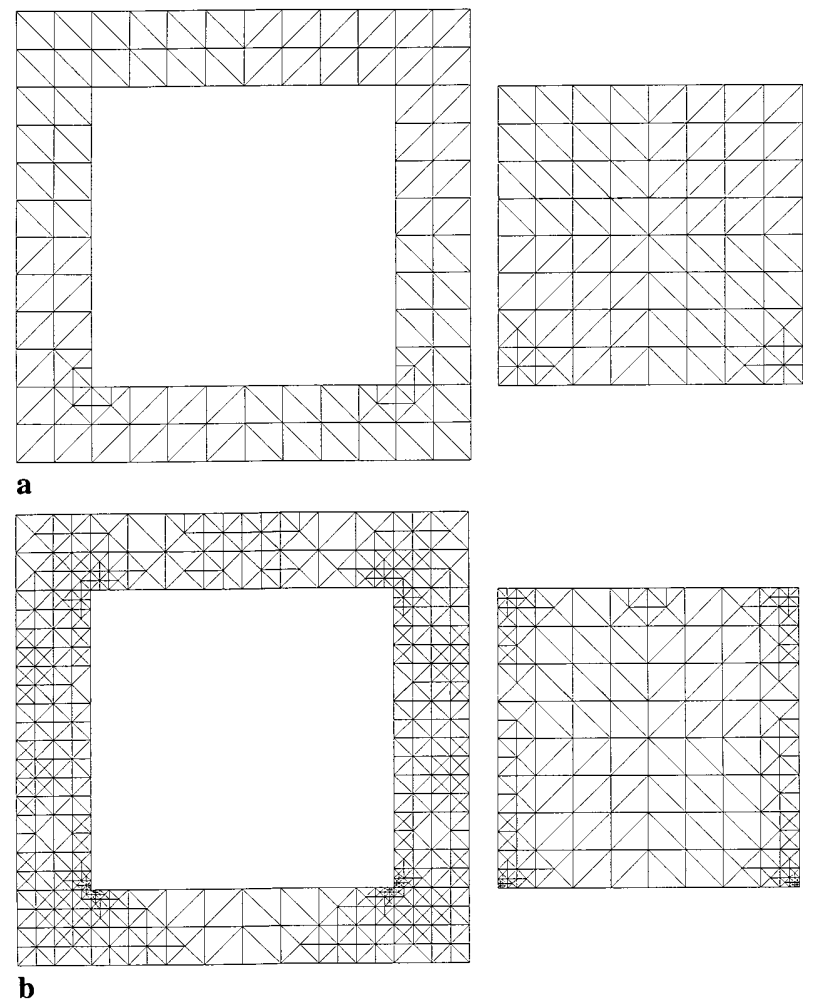

Fig. 5a,b. Some refined triangulations for Eigenmode $F_{01}$. a First refinement step for the solid and fluid domains, b Fifth refinement step for the solid and fluid domains

We present above the results obtained with meshes generated by the adaptive refinement procedure defined in the previous section and using the same simple refinement strategy as in the first problem.

Table 7 to Table 12 show the results obtained in six steps of the refinement procedure for the structural eigenmodes $S_{1}, S_{2}$ and $S_{3}$ and for the fluid eigenmodes $F_{01}, F_{10}$ and $F_{11}$, respectively.

Again, we remark the reduction of the necessary computational effort to achieve a given accuracy.

Finally, Figs. 4 and 5 show some refined meshes for eigenmodes $S_{1}$ and $F_{01}$, respectively.

\section{References}

Babuška I, Rheinboldt WC (1978) Error estimates for adaptive finite element computations. SIAM J. Num. Anal. 15:736-754

Babuška I, Rheinboldt WC (1978) A posteriori error estimates for the finite element method. Int. J. Numer. Meth. Eng. 12:15971615
Bermúdez A, Durán R, Muschietti MA, Rodriguez R, Solomin J (1995) Finite element vibration analysis of fluid-solid systems without spurious modes. SIAM J. Numer. Anal. 32:1280-1295

Bermúdez A, Durán R, Rodriguez R (1997) Finite element solution of incompressible fluid-structure vibration problems. Int. J. Numer. Meth. Eng. 40:1435-1448

Bermúdez A, Durán R, Rodriguez R (1998) Finite element analysis of compressible and incompressible fluid-solid systems. Math. Comp. 67:111-136

Bermúdez A, Rodriguez R (1994) Finite element computation of the vibration modes of a fluid-solid system. Comput. Methods Appl. Mech. Eng. 119:355-370

Boujot J (1987) Mathematical formulation of fluid-structure interaction problems. $M^{2} A N$ 21:239-260

Brezzi F, Fortin M (1991) Mixed and Hybrid Finite Element Methods. New York: Springer-Verlag

Clement P (1975) Approximation by finite element functions using local regularization. R.A.I.R.O. Anal. Numer. 9:77-84

Chen HC, Taylor RL (1990) Vibration analysis of fluid-solid systems using a finite element displacement formulation. Inter. J. Numer. Meth. Eng. 29:683-698

Dautray R, Lions J-L (1990) Mathemathical Analysis and Numerical Methods for Science and Technology, vol. 4, pp. 317331. Berlin Heidelberg New York London Paris Tokyo Hong Kong, Springer-Verlag

Hamdi M, Ousset Y, Verchery G (1978) A displacement method for the analysis of vibrations of coupled fluid-structure systems. Inter. J. Numer. Meth. Eng. 13:139-150

Morand HJ-P, Ohayon R (1995) Fluid Structure Interaction. Chichester New York Brisbane Toronto Singapure, John Wiley \& Sons

Raviart PA, Thomas J (1977) A mixed finite element method for second order elliptic problems, Mathematical aspects of the Finite Elements Method, Lecture Notes in Math. 606, pp. 292315. New York, Springer-Verlag

Rannacher R, Suttmeier FT (1997) A feed-back approach to error control in finite element methods: application to linear elasticity. Comp. Mech. 19:434-446

Ruge P (1996) Hybrid time-finite-elements with time-step adaptation by discontinuity control. Comp. Mech. 17:392-397

Smolinski P, Sleith S, Belytschko T (1996) Stability of an explicit multi-time step integration algorithn for linear structural dynamics equations. Comp. Mech. 18:236-244

Rodriguez R, Solomin J (1996) The order of convergence of eigenfrequencies in finite element approximations of fluid structure interaction problems. Numer. Math. 55:309-325

Verfürth R (1989) A posteriori error estimators for the Stokes Equations. Math. Comp. 62:445-475

Verfürth R (1994) A posteriori error estimates for nonlinear problems. Math. Comp. 62:445-475

Verfürth R (1994) A posteriori error estimation and adaptive mesh-refinement techniques. J. Comput. Appl. Math. 50:67-83

Zienkiewicz OC, Zhu JZ (1987) A simple error estimator and adaptive procedure for practical engineering analysis. J. Numer. Methods Engrg. 24:337-357 